\title{
The Effects of Hydrogen Bonding on the Acidity of Uracil
}

Mariana Di Laudo, Sarah R. Whittleton and Stacey D. Wetmore*

Department of Chemistry, Mount Allison University, 63C York Street, Sackville, New

Brunswick, E4L 1G8, Canada

Supporting Information 


\section{(Neutral) Uracil-Water Complexes}

\section{O2(N3)}

7
6
8
8
8
6
6
1
1
6
1
1
1
1
7

\section{4(N3)}

\section{O4(C5)}

1

$\begin{array}{cc}0.78366 & -1.62854 \\ -0.4196 & -0.93705 \\ -1.49927 & -1.5294 \\ -3.14007 & 0.73597 \\ 0.89655 & 2.39023 \\ 0.91858 & 1.16838 \\ 2.12249 & 0.34001 \\ 3.08296 & 0.83643 \\ 2.87108 & -1.66904 \\ 2.01327 & -1.00598 \\ 0.70203 & -2.6356 \\ -1.16657 & 0.95721 \\ -2.97004 & -0.22251 \\ -3.86987 & 0.9457 \\ -0.28591 & 0.43158\end{array}$

0.00151

0.00943

0.01884

$-0.09347$

0.0127

0.00438

$-0.00838$

$-0.0162$

$-0.01866$

$-0.0097$

$-0.00156$

0.00994

$-0.02428$

0.5004

0.00868

$$
\begin{array}{cc}
0.82629 & -0.01135 \\
0.32118 & -0.0051 \\
-1.5446 & -0.01333 \\
-1.05136 & -0.00659 \\
-2.8106 & -0.00059 \\
-1.73037 & 0.00056 \\
-0.99415 & 0.00767 \\
-1.51648 & 0.01549 \\
0.40024 & 0.00619 \\
0.94168 & 0.01062 \\
2.34078 & 0.01056 \\
1.12308 & 0.0039 \\
-0.19631 & -0.02593 \\
0.76223 & -0.08937 \\
0.95817 & 0.50617
\end{array}
$$

$\begin{array}{lll}0.4986 & 0.05963 & -2.08938 \\ 0.53247 & 0.01923 & -1.00836 \\ 1.69558 & -0.04425 & -0.32101 \\ 2.6631 & -0.0592 & -0.81003 \\ 1.72772 & -0.09431 & 1.05432 \\ 2.60248 & -0.14208 & 1.55826 \\ 0.58741 & -0.08427 & 1.85173 \\ 0.62808 & -0.12902 & 3.06974 \\ -0.58753 & -0.01856 & 1.11828 \\ -1.44447 & -0.00948 & 1.66071 \\ -0.72496 & 0.03508 & -0.27663 \\ -1.85119 & 0.09001 & -0.7726 \\ -1.79174 & 0.14422 & -2.67255 \\ -1.39371 & 0.16012 & -3.56454 \\ -2.11766 & 0.27326 & -4.19045\end{array}$




\section{(Neutral) Uracil-Ammonia Complexes}

\section{O2(N3)}

7
6
8
7
1
8
6
6
1
1
6
1
1
1
1
7

\section{4(N3)}

1

1

7

1

8

6

7

1

8

6

7

1

1

6

6

1

\section{O4(C5)}

$\begin{array}{rrr}-1.16043 & -1.49032 & 0.00012 \\ 0.17669 & -1.09625 & -0.00026 \\ 1.08808 & -1.91847 & -0.00034 \\ 3.25733 & 0.44349 & 0.00044 \\ 3.75632 & 0.7766 & 0.82086 \\ -0.33783 & 2.44589 & -0.00024 \\ -0.63663 & 1.25775 & -0.00016 \\ -2.0024 & 0.73504 & 0.00025 \\ -2.82045 & 1.44208 & 0.00047 \\ -3.19993 & -1.04366 & 0.00067 \\ -2.21037 & -0.59986 & 0.00037 \\ -1.31461 & -2.48863 & 0.00027 \\ 1.35781 & 0.56971 & -0.0007 \\ 3.2805 & -0.57517 & 0.00216 \\ 3.75695 & 0.77372 & -0.82076 \\ 0.36277 & 0.26768 & -0.00049\end{array}$

3.75419

3.74132

3.23722

3.21777

1.22618

0.22942

0.36346

1.33997

$-0.48598$

$-0.65374$

$-1.93317$

$-2.69339$

$-3.20853$

$-2.16527$

$-1.14804$

$-1.31973$
$-0.81646$

0.82559

$-0.00072$

$-0.00516$

0.00177

$-0.00004$

$-0.00201$

$-0.00219$

0.00093

$-0.00016$

0.00027

0.00153

$-0.00069$

$-0.00031$

$-0.00054$

$-0.00067$
0.32768

0.43859

1.64292

2.57883

1.76361

2.66914

0.67622

0.79687

$-0.5432$

$-1.36346$

$-0.77368$

$-1.92607$

$-1.93569$

$-1.45284$

$-1.76682$

$-1.75333$
$-0.00497$

$-0.00466$

$-0.01445$

$-0.02321$

$-0.01399$

$-0.02133$

$-0.00359$

$-0.0033$

0.0062

0.01401

0.00679

0.01642

0.0128

0.00717

$-0.80968$

0.82718
$-2.06648$

$-0.98766$

$-0.3736$

$-0.92118$

0.99951

1.44781

1.86692

3.08163

1.2097

1.80592

$-0.17899$

$-0.60443$

$-2.83753$

$-3.73698$

$-4.2544$

$-4.25741$ 


\section{(Neutral) Uracil-Hydrogen Fluoride Complexes}

\section{O2(N3)}

7
6
8
9
8
6
6
1
1
6
1
1
1
7

\section{O4(N3)}

\section{O4(C5)}

$$
\begin{array}{rr}
-1.66173 & 0.00019 \\
-0.80455 & 0.00051 \\
-1.23859 & -0.00043 \\
0.84832 & -0.00042 \\
2.298 & -0.00041 \\
1.08768 & 0.00021 \\
0.09165 & -0.00047 \\
0.44236 & -0.00132 \\
-2.00516 & -0.00055 \\
-1.22372 & -0.00027 \\
-2.64676 & -0.00004 \\
1.17767 & 0.00107 \\
-0.04329 & -0.00153 \\
0.52726 & 0.00166
\end{array}
$$

$-2.77375$

$-0.21526$

$$
\begin{array}{r}
-2.97703 \\
-2.04333 \\
-2.72357 \\
-1.83091 \\
-0.40211 \\
-0.58149 \\
0.55869 \\
1.74106 \\
0.25074 \\
1.03784 \\
-1.22635 \\
-1.01568 \\
2.75717 \\
3.10605
\end{array}
$$

$$
\begin{array}{r}
0.50054 \\
0.11257 \\
-1.85764 \\
-1.24218 \\
-2.83055 \\
-1.76484 \\
-0.87091 \\
-1.2482 \\
0.48887 \\
1.13996 \\
2.25561 \\
1.05742 \\
-0.04452 \\
0.86066
\end{array}
$$

0.00006

$-0.00079$

0.00006

$-0.0001$

0.00061

0.0003

0.00012

0.00022

$-0.00018$

0.00002

0.00059

0.00002

0.00055

$-0.00034$

$\begin{array}{lll}0.6158 & -0.00137 & -2.1116 \\ 0.6105 & -0.00026 & -1.02994 \\ 1.75018 & 0.00147 & -0.29935 \\ 2.73487 & 0.00206 & -0.75303 \\ 1.7309 & 0.00271 & 1.07443 \\ 2.58739 & 0.00386 & 1.6118 \\ 0.56079 & 0.00262 & 1.82953 \\ 0.55445 & 0.0038 & 3.04695 \\ -0.58859 & 0.00099 & 1.04949 \\ -1.46636 & 0.00034 & 1.55851 \\ -0.66727 & -0.00061 & -0.34304 \\ -1.78129 & -0.00219 & -0.88223 \\ -1.77689 & -0.0049 & -2.49361 \\ -1.60015 & -0.00645 & -3.43783\end{array}$




\section{(Neutral) Uracil O2(N3)-O4(N3) Complexes}

$\begin{array}{cccc}\mathbf{N H}_{\mathbf{3}}-\mathbf{N H}_{3} & & & \\ 1 & -1.49996 & -0.10712 & -3.3636 \\ 6 & -1.34246 & -0.06326 & -2.29472 \\ 6 & 0.03242 & -0.07845 & -1.79646 \\ 7 & 0.1526 & -0.01848 & -0.39556 \\ 1 & 1.13345 & -0.02865 & -0.00161 \\ 7 & 2.78984 & -0.05329 & 0.69067 \\ 1 & 3.34885 & 0.73251 & 0.36778 \\ 1 & 2.71429 & -0.00684 & 1.71462 \\ 1 & 3.29255 & -0.90038 & 0.43755 \\ 1 & 1.83605 & 0.93938 & 4.1619 \\ 1 & 1.76943 & -0.69444 & 4.21565 \\ 7 & 1.76281 & 0.10431 & 3.58675 \\ 1 & 0.85665 & 0.12475 & 3.11468 \\ 8 & -0.73932 & 0.10003 & 1.72908 \\ 6 & -0.87782 & 0.04972 & 0.50721 \\ 7 & -2.14982 & 0.05928 & -0.06353 \\ 1 & -2.91481 & 0.1092 & 0.5942 \\ 6 & -2.37007 & 0.00415 & -1.42101 \\ 1 & -3.41011 & 0.01877 & -1.72816 \\ 8 & 1.03098 & -0.13789 & -2.5055\end{array}$

\section{$\mathrm{NH}_{3}-\mathrm{H}_{2} \mathrm{O}$}

7

6

8

\section{$\mathrm{NH}_{3}-\mathrm{HF}$}

$\begin{array}{ccc}1.32759 & -0.15853 & -2.1218 \\ 1.50978 & -0.22593 & -0.73697 \\ 2.61376 & -0.41469 & -0.24738 \\ 0.66817 & -0.20096 & 2.82258 \\ 1.05726 & -1.08971 & 3.12667 \\ -1.90412 & 0.28531 & 0.2245 \\ -0.92646 & 0.15185 & -0.52097 \\ -1.01393 & 0.20429 & -1.97418 \\ -1.98265 & 0.36919 & -2.42473 \\ 0.10838 & 0.07896 & -3.79844 \\ 0.10741 & 0.049 & -2.71429 \\ 2.1685 & -0.2756 & -2.66976 \\ 0.46753 & -0.11214 & 1.0457 \\ 1.27463 & 0.53222 & 3.18115 \\ -0.25815 & -0.08785 & 3.24193 \\ 0.35001 & -0.06251 & -0.00471 \\ -2.17422 & 0.3496 & 2.02226 \\ -2.21129 & 0.36733 & 3.00194 \\ -3.12922 & 0.21059 & 3.24877\end{array}$

$\begin{array}{rcc}-0.17829 & -0.021 & 0.2955 \\ 0.00432 & -0.10749 & 1.66471 \\ 1.34328 & -0.09405 & 2.06581 \\ 2.39339 & -0.00253 & 1.18959 \\ 2.18648 & 0.08118 & -0.14523 \\ 0.82664 & 0.0739 & -0.65506 \\ -0.90668 & -0.18928 & 2.4732 \\ 0.56887 & 0.14852 & -1.86889 \\ -2.86902 & -0.05143 & -0.57336\end{array}$




$\begin{array}{ccc}-3.36176 & -0.9028 & -0.31698 \\ 3.00046 & 0.15392 & -0.85246 \\ 3.38235 & -0.00224 & 1.63455 \\ 1.4912 & -0.15597 & 3.0638 \\ -1.19072 & -0.0297 & -0.02278 \\ -3.41831 & 0.73311 & -0.23065 \\ -2.8235 & 0.00534 & -1.59246 \\ -0.74666 & 0.14435 & -2.67776 \\ -1.57416 & 0.14167 & -3.1866\end{array}$

\section{$\mathrm{H}_{2} \mathrm{O}-\mathrm{NH}_{3}$}

1.3122
0.08918

$-0.96366$

$-3.64565$

1. 3075

1. 35712

2. 58969

3. 53302

3. 40013

2. 52355

1. 25645

$-0.73716$

$-2.73962$

$-4.24707$

0.17196

$-2.36216$

$-2.28469$

$-3.05347$

$-2.38927$

$$
\begin{array}{r}
-1.66241 \\
-1.00119 \\
-1.64193 \\
-0.79927 \\
2.33975 \\
1.11899 \\
0.33319 \\
0.85841 \\
-1.64936 \\
-1.01164 \\
-2.66816 \\
0.90123 \\
-1.16751 \\
-1.5077 \\
0.36073 \\
2.44144 \\
1.80018 \\
1.12781 \\
2.34982
\end{array}
$$

-0.08134
-0.05968
-0.12896
-0.25513
0.21593
0.12495
0.0932
0.15229
-0.03615
-0.00759
-0.15855
0.06138
-0.21012
-0.00084
0.04146
-0.69521
0.09009
0.03683
0.93902

\section{$\mathrm{H}_{2} \mathrm{O}-\mathrm{H}_{2} \mathrm{O}$}

$$
\begin{aligned}
& -2.49295 \\
& -1.33906 \\
& -1.39246 \\
& 2.28363 \\
& 1.05859 \\
& -0.04884 \\
& -1.30835 \\
& -1.27684 \\
& -3.4433 \\
& -2.47364 \\
& -3.3614 \\
& 0.70939 \\
& 2.4849 \\
& 2.96574 \\
& -0.16334 \\
& 2.75791 \\
& 3.6198
\end{aligned}
$$$$
4.22051
$$$$
0.27136
$$$$
1.06003
$$$$
2.2783
$$$$
\text { 1. } 72832
$$$$
-1.60851
$$$$
-1.05774
$$$$
-1.786
$$$$
-2.86624
$$$$
-1.58531
$$$$
-1.09985
$$$$
0.78685
$$$$
0.89473
$$$$
\text { 2. } 47295
$$$$
1.0339
$$$$
0.3315
$$$$
-1.06251
$$$$
-0.5966
$$$$
-1.00511
$$

\section{$\mathrm{H}_{2} \mathrm{O}-\mathrm{HF}$}

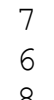

6
8

$\begin{array}{lll}1.1787 & 0.00303 & -2.16941 \\ 1.48138 & 0.0067 & -0.80559 \\ 2.62896 & 0.02793 & -0.39735\end{array}$




$\begin{array}{rcr}0.72968 & -0.19345 & 2.79224 \\ -1.89839 & -0.01443 & 0.41623 \\ -0.95933 & -0.01606 & -0.39938 \\ -1.17162 & -0.02105 & -1.83391 \\ -2.18707 & -0.02994 & -2.20363 \\ -0.19084 & -0.0142 & -3.74185 \\ -0.10013 & -0.01209 & -2.66133 \\ 1.98098 & 0.01474 & -2.78446 \\ 0.56634 & -0.04186 & 1.0358 \\ 1.3532 & 0.3257 & 3.31301 \\ -0.16706 & -0.03951 & 3.15122 \\ 0.36355 & -0.01236 & 0.01638 \\ -1.94216 & 0.08893 & 1.92199 \\ -1.93256 & 0.16179 & 2.89906\end{array}$

\section{HF-NH}

$$
\begin{array}{r}
1.08411 \\
0.89879 \\
1.88654 \\
2.10607 \\
-2.65243 \\
-1.53207 \\
-1.23762 \\
-2.06574 \\
0.32689 \\
0.04266 \\
2.04515 \\
-0.5778 \\
2.0332 \\
-0.39592 \\
-1.40008 \\
-0.83385 \\
0.08652 \\
-1.2866
\end{array}
$$

\section{HF- $\mathrm{H}_{2} \mathrm{O}$}

$$
\begin{array}{rr}
-0.07105 & -1.77489 \\
-0.05979 & -0.40295 \\
-0.1262 & 0.34492 \\
-0.13859 & 2.86702 \\
0.17459 & -0.33715 \\
0.10095 & -0.82364 \\
0.08163 & -2.25514 \\
0.13602 & -2.94832 \\
-0.02146 & -3.7208 \\
-0.00276 & -2.67475 \\
-0.13593 & -2.08082 \\
0.03643 & 1.05773 \\
-0.13375 & 1.90321 \\
0.02655 & 0.01313 \\
-0.72509 & 3.13239 \\
0.05616 & 2.81178 \\
-0.00689 & 3.24938 \\
0.90893 & 3.13072
\end{array}
$$

$$
\begin{array}{rcr}
1.20453 & -0.08436 & -1.67349 \\
0.91072 & -0.06974 & -0.32372 \\
1.83471 & -0.11837 & 0.50423 \\
1.78098 & 0.12178 & 2.99825 \\
-2.63732 & 0.15606 & -0.54349 \\
-1.48588 & 0.07577 & -0.94182 \\
-1.07273 & 0.05025 & -2.34293 \\
-1.84118 & 0.09699 & -3.10215 \\
0.60674 & -0.05032 & -3.67451 \\
0.23776 & -0.02848 & -2.65549 \\
2.18782 & -0.1359 & -1.9016 \\
-0.67443 & -0.01829 & 0.98901 \\
1.83099 & 0.02414 & 2.03051 \\
-0.41568 & 0.00011 & -0.0167 \\
-1.55044 & 0.3655 & 3.2357 \\
-0.90011 & -0.15503 & 2.75014 \\
-0.02777 & -0.01649 & 3.16695
\end{array}
$$




$\begin{array}{crrr}\text { HF-HF } & & & \\ 7 & 1.18125 & 0.00095 & -1.81899 \\ 6 & 1.17209 & 0.00075 & -0.43529 \\ 8 & 2.22488 & 0.00154 & 0.20194 \\ 9 & 1.90442 & 0.00396 & 2.76453 \\ 8 & -2.37591 & -0.00068 & 0.03928 \\ 6 & -1.30025 & -0.00025 & -0.56881 \\ 6 & -1.18607 & -0.00078 & -2.01445 \\ 1 & -2.09494 & -0.00146 & -2.5994 \\ 1 & 0.19409 & 0 . & -3.65719 \\ 6 & 0.03977 & 0.00001 & -2.58437 \\ 1 & 2.10026 & 0.00142 & -2.24158 \\ 1 & -0.13512 & -0.00205 & 1.15498 \\ 1 & 2.17354 & 0.00184 & 1.85151 \\ 7 & -0.08394 & -0.00003 & 0.13014 \\ 1 & -2.21552 & -0.00032 & 1.64281 \\ 9 & -1.77647 & -0.0052 & 2.49754\end{array}$

\section{(Neutral) Uracil O4(N3)-O4(C5) Complexes}

\section{$\mathbf{N H}_{3}-\mathbf{N H}_{3}$}

$\begin{array}{lccc}6 & -0.55808 & 0.00091 & -0.01912 \\ 6 & -0.5662 & 0.00125 & 1.4378 \\ 6 & 0.61255 & 0.00031 & 2.10094 \\ 7 & 1.81628 & -0.00106 & 1.43543 \\ 6 & 1.92712 & -0.00162 & 0.04407 \\ 7 & 0.7106 & -0.00056 & -0.60935 \\ 8 & 3.01269 & -0.00277 & -0.5179 \\ 8 & -1.5696 & 0.00172 & -0.73053 \\ 7 & -3.94084 & 0.00231 & 1.3366 \\ 1 & -1.5239 & 0.00241 & 1.94533 \\ 1 & 0.67691 & 0.00061 & 3.18364 \\ 1 & 2.69476 & -0.00172 & 1.93448 \\ 1 & 0.72854 & -0.0009 & -1.64891 \\ 1 & -3.39762 & 0.00822 & 0.47177 \\ 1 & -4.55038 & 0.81578 & 1.33127 \\ 1 & -4.53576 & -0.8219 & 1.32829 \\ 1 & -0.91661 & 0.00068 & -3.08792 \\ 7 & 0.04589 & -0.00046 & -3.42481 \\ 1 & 0.18084 & -0.8215 & -4.00872 \\ 1 & 0.1827 & 0.82007 & -4.00899\end{array}$

\section{$\mathrm{NH}_{3}-\mathrm{H}_{2} \mathrm{O}$}

$\begin{array}{rrr}-0.59499 & -0.00068 & 0.03732 \\ -0.58893 & 0.00152 & 1.49216 \\ 0.59732 & 0.00078 & 2.1427 \\ 1.79148 & -0.00195 & 1.46327 \\ 1.8856 & -0.00404 & 0.07016 \\ 0.66058 & -0.00345 & -0.56881 \\ 2.96316 & -0.00621 & -0.5055 \\ -1.61609 & -0.0001 & -0.66607 \\ -3.80667 & 0.02004 & 1.10119 \\ -1.53604 & 0.00313 & 2.01575 \\ 0.67313 & 0.00233 & 3.22449 \\ 2.67599 & -0.00219 & 1.95192\end{array}$




1
1
1
1
7
1
1

\section{$\mathbf{N H}_{3}-\mathbf{H F}$}

6
6
6
7
6
7
8
8
9
1
1
1
1
1
1
7
1
1

\section{$\mathrm{H}_{2} \mathrm{O}-\mathrm{NH}_{3}$}

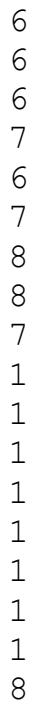

\section{$\mathrm{H}_{2} \mathrm{O}-\mathrm{H}_{2} \mathrm{O}$}

6
6
6
7
6

$$
\begin{array}{r}
0.66523 \\
-3.18509 \\
-4.69283 \\
-0.91804 \\
0.07248 \\
0.26721 \\
0.26143
\end{array}
$$

$-0.61958$

$-0.60211$

0.59262

1.7755

1.85194

0.6175

2.92008

$-1.65181$

$-3.79702$

$-1.54329$

0.68107

2.66658

0.60661

$-3.02308$

$-0.90541$

0.10582

0.34677

0.36883

$-0.49904$

$-0.52148$

0.65206

1. 86152

1.98849

0.77267

3.07345

$-1.50957$

$-3.86415$

$-1.48557$

0.70565

2.73488

0.80872

$-3.37584$

$-4.45113$

$-4.48058$

$-1.05182$

$-0.31665$

$-0.49265$

$$
\begin{array}{rr}
-0.00391 & -1.61122 \\
0.01091 & 0.34507 \\
-0.06556 & 0.73261 \\
0.00262 & -3.15241 \\
-0.00057 & -3.39139 \\
0.82039 & -3.9583 \\
-0.82132 & -3.96054
\end{array}
$$

$$
\begin{array}{cc}
0.00143 & 0.09983 \\
0.00858 & 1.55067 \\
0.00826 & 2.18803 \\
0.00128 & 1.49355 \\
-0.00628 & 0.09821 \\
-0.00584 & -0.52539 \\
-0.01241 & -0.49172 \\
0.00136 & -0.59749 \\
0.01814 & 0.75475 \\
0.01419 & 2.08361 \\
0.01351 & 3.2688 \\
0.00088 & 1.97091 \\
-0.01123 & -1.57291 \\
0.0117 & 0.17622 \\
-0.02826 & -3.22116 \\
-0.01459 & -3.34347 \\
0.8134 & -3.88219 \\
-0.82694 & -3.89554
\end{array}
$$

$\begin{array}{rc}-0.01498 & -0.06611 \\ 0.02041 & 1.3861 \\ 0.04851 & 2.05911 \\ 0.04233 & 1.40608 \\ 0.00753 & 0.01789 \\ -0.01633 & -0.64535 \\ -0.00212 & -0.53931 \\ -0.04301 & -0.78487 \\ 0.02586 & 1.34068 \\ 0.02539 & 1.88229 \\ 0.07705 & 3.14186 \\ 0.06223 & 1.91421 \\ -0.03809 & -1.67052 \\ -0.00059 & 0.44496 \\ 0.85579 & 1.35253 \\ -0.78122 & 1.386 \\ -0.04783 & -2.646 \\ 0.01217 & -3.2887 \\ -0.62093 & -3.99404\end{array}$

$\begin{array}{ccc}-0.5273 & -0.00805 & -0.01334 \\ -0.54085 & 0.01668 & 1.43729 \\ 0.63784 & 0.03302 & 2.1028 \\ 1.8408 & 0.02434 & 1.44117 \\ 1.95734 & -0.0026 & 0.05143\end{array}$ 


$\begin{array}{crr}0.73518 & -0.01449 & -0.60247 \\ 3.0365 & -0.01579 & -0.51484 \\ -1.544 & -0.02361 & -0.72927 \\ -3.72389 & 0.03408 & 1.07896 \\ -1.49561 & 0.02248 & 1.94675 \\ 0.6981 & 0.05347 & 3.18524 \\ 2.71796 & 0.03564 & 1.94337 \\ 0.76111 & -0.02973 & -1.62892 \\ -3.15018 & 0.01392 & 0.28886 \\ -4.63333 & -0.03234 & 0.76722 \\ -1.09152 & -0.02614 & -2.62908 \\ -0.33268 & 0.03557 & -3.24099\end{array}$

$-0.48798$

$-0.58256-3.96437$

\section{$\mathrm{H}_{2} \mathrm{O}-\mathrm{HF}$}

6
6
6
7
6
7
8
8
9
1
1
1
1
1
1
8
1

-0.54322
-0.54787
0.63748
1.83109
1.93418
0.70391
3.00564
-1.56973
-3.73401
-1.49717
0.70705
2.71385
0.71565
-2.96861
-1.13683
-0.32442
-0.44814

0.00275

0.05139

0.06971

0.0407

$-0.0103$

$-0.02369$

$-0.04109$

$-0.0165$

$-0.00281$

0.0731

0.1076

0.0534

$-0.05547$

$-0.00494$

$-0.04546$

0.0115

$-0.53467$

$\begin{array}{ccc}-0.45513 & -0.00193 & -0.11137 \\ -0.50006 & -0.00292 & 1.33481 \\ 0.66738 & -0.00157 & 2.02117 \\ 1.88346 & 0.0008 & 1.38285 \\ 2.02872 & 0.00199 & -0.0028 \\ 0.81497 & 0.00041 & -0.67949 \\ 3.11419 & 0.00411 & -0.55365 \\ -1.46502 & -0.00289 & -0.84329 \\ -3.80524 & -0.00075 & 1.35818 \\ -1.47284 & -0.00477 & 1.81569 \\ 0.70814 & -0.00226 & 3.1047 \\ 2.7506 & 0.00183 & 1.90278 \\ 0.86884 & 0.00137 & -1.69938 \\ -3.41644 & -0.01339 & 0.41561 \\ -4.38829 & 0.82806 & 1.44148 \\ -4.41516 & -0.80863 & 1.45348 \\ -1.10397 & -0.0004 & -2.365 \\ -0.60246 & 0.00131 & -3.19727\end{array}$

0.05009

1. 49658

2.15273

1.47997

0.08841

$-0.55359$

$-0.48811$

$-0.66089$

0.69809

2.01437

3.2341

1.973

$-1.58168$

0.1161

$-2.64665$

$-3.18073$

$-3.9656$

\section{HF-NH 3}




$\begin{array}{cccr}\mathbf{H F}-\mathbf{H}_{2} \mathbf{O} & & & \\ 6 & -0.48156 & -0.0004 & -0.05514 \\ 6 & -0.51678 & -0.00122 & 1.38951 \\ 6 & 0.65606 & -0.00105 & 2.06819 \\ 7 & 1.86555 & -0.00022 & 1.42142 \\ 6 & 2.00075 & 0.0003 & 0.03401 \\ 7 & 0.78059 & 0.00007 & -0.63303 \\ 8 & 3.08065 & 0.00082 & -0.52551 \\ 8 & -1.4966 & -0.00008 & -0.78459 \\ 8 & -3.66818 & 0.00851 & 1.07686 \\ 1 & -1.47876 & -0.00224 & 1.88565 \\ 1 & 0.70305 & -0.00155 & 3.15135 \\ 1 & 2.73658 & 0.00003 & 1.93518 \\ 1 & 0.82569 & 0.0003 & -1.65353 \\ 1 & -3.16686 & 0.00314 & 0.24203 \\ 1 & -4.60236 & -0.03789 & 0.84391 \\ 1 & -1.14238 & -0.0006 & -2.32844 \\ 9 & -0.63063 & -0.00221 & -3.14982\end{array}$

\section{HF-HF}

6

6

6

7

6

7

8

8

9

1

1

1

1

1

9
$-0.49805$

$-0.51888$

0.66242

1.86102

1.97947

0.74935

3.05039

$-1.52551$

$-3.68151$

$-1.47331$

0.72097

2.73899

0.78001

$-2.97003$

$-1.18293$

$-0.6335$
0.00033

0.00013

$-0.00015$

$-0.0003$

$-0.00014$

0.00007

$-0.00041$

0.00054

0.00048

0.00024

$-0.00028$

$-0.00053$

$-0.00013$

0.00033

0.00066

$-0.00057$
0.0063
1.44798
2.11453
1.45345
0.06299
$-0.58939$
$-0.50986$
$-0.71437$
0.72812
1.95667
3.19712
1.9561
$-1.61148$
0.09221
$-2.30891$
$-3.0978$

\section{(Neutral) Uracil 02(N3)-O4(C5) Complexes}

$\mathbf{N H}_{\mathbf{3}}-\mathbf{N H}_{\mathbf{3}}$
7
6
8
7
1
8
6
6
1
1
6
1
1
1
1

$$
\begin{gathered}
0.01444 \\
-0.00211 \\
-0.01102 \\
-0.0432 \\
-0.87108 \\
-0.00522 \\
0.0015 \\
0.01847 \\
0.02587 \\
0.03652 \\
0.02412 \\
0.01814 \\
-0.02004 \\
-0.04065 \\
0.77033
\end{gathered}
$$

-1.61652
-0.25331
0.17106
3.17256
3.76107
0.95569
0.1266
-1.32147
-1.70399
-3.20933
-2.12717
-2.22087
1.57024
2.67625
3.7811 
$-3.63999 \quad 0.01149$

0.55007

$-4.28946$

0.02204

$-0.00354$

$-4.88799$

$-0.79606$

$-0.79206$

$-4.88423$

0.8409

$-0.71499$

\section{$\mathrm{NH}_{3}-\mathrm{H}_{2} \mathrm{O}$}

$-0.0927$

$-1.63672$

1.66753

$-0.09137$

$-0.26769$

2.80784

$-0.14744$

0.18022

1.63649

$-0.05285$

3.18337

1.50004

$-0.86123$

3.78436

$-1.71261$

0.10082

0.8798

$-0.78718$

0.04295

0.06415

$-0.94987$

0.03591

$-1.38442$

$-1.95133$

0.08503

$-1.79194$

0.09523

$-0.04011$

$-3.25402$

0.14748

$-0.0311$

$-2.17097$

2.23366

$-0.14313$

0.71255

$-0.02074$

$-2.22601$

2. 57175

$-0.10696$

1. 58756

0.53329

0.7777

1.54088

2.7828

$-0.02221$

3.76738

$-3.3889$

0.17777

0.51441

$-4.0938$

0.20692

0.0678

$-4.92919$

0.28522

$-0.611$

$-0.13722$

\section{$\mathbf{N H}_{3}-\mathbf{H F}$}

\section{7}

6

8

7

1

8

6

1

1

6

1
1

1

1

7

1

9

\section{$\mathrm{H}_{2} \mathrm{O}-\mathrm{NH}_{3}$}

$$
\begin{array}{r}
1.43151 \\
1.6068 \\
2.72311 \\
1.75123 \\
2.03784 \\
-1.83813 \\
-0.86615 \\
-0.94505 \\
-1.92249 \\
0.21278 \\
0.19933 \\
2.28613 \\
0.51188 \\
2.33531 \\
1.97917 \\
0.42189 \\
-3.27441 \\
-4.04977
\end{array}
$$

0.05371

$-0.01601$

$-0.04465$

$-0.06999$

$-0.60534$

$-0.04634$

$-0.01431$

0.05772

0.08529

0.14323

0.08899

0.07513

$-0.09377$

$-0.35874$

0.90307

$-0.04706$

0.00024

0.03598

$-1.56847$

$-0.19163$

0.31683

3.17746

3. 99452

0.75769

$-0.00842$

$-1.45754$

$-1.92018$

$-3.25985$

$-2.17716$

$-2.10816$

1.55185

2. 39527

3. 36896

0.52365

0.05948

$-0.5109$

-0.07109
0.00659
0.04517
0.30162
0.01391
-0.01171
-0.0896

$-1.48118$

$-0.11282$

0.37201

3. 04729

0.9174

0.13582

$-1.31775$ 


\begin{tabular}{rrr}
-1.93885 & -0.12434 & -1.75349 \\
0.17259 & -0.17251 & \multicolumn{1}{c}{-3.14351} \\
0.18119 & -0.11545 & \multicolumn{1}{c}{-2.0608} \\
2.27396 & -0.08851 & -2.04055 \\
0.6049 & 0.09582 & 1.64368 \\
2.64416 & 0.19673 & 2.31826 \\
2.30762 & -0.24256 & 3.78247 \\
0.47448 & 0.03377 & 0.62845 \\
-3.72322 & -0.05942 & -0.17104 \\
-4.27483 & -0.10747 & -1.02892 \\
-4.87559 & -0.92535 & -0.96805 \\
-4.87983 & 0.70912 & -1.05636
\end{tabular}

\section{$\mathrm{H}_{2} \mathrm{O}-\mathrm{H}_{2} \mathrm{O}$}

$\begin{array}{rrr}1.46424 & -0.1131 & -1.46969 \\ 1.61233 & -0.07142 & -0.09235 \\ 2.72129 & -0.09947 & 0.44043 \\ 1.82329 & -0.08601 & 3.09203 \\ -1.8431 & 0.11925 & 0.79927 \\ -0.8662 & 0.04441 & 0.0534 \\ -0.91525 & -0.00692 & -1.40198 \\ -1.88397 & 0.01731 & -1.88449 \\ 0.27082 & -0.12483 & -3.18286 \\ 0.23906 & -0.08346 & -2.10003 \\ 2.32589 & -0.17154 & -1.99459 \\ 0.51515 & 0.03163 & 1.62301 \\ 2.51808 & -0.06468 & 2.40743 \\ 2.09967 & 0.49827 & 3.80727 \\ 0.42391 & 0.00415 & 0.60115 \\ -3.47385 & 0.17538 & -0.15616 \\ -4.08076 & 0.19019 & -0.9223 \\ -4.97426 & 0.11378 & -0.56945\end{array}$

\section{$\mathrm{H}_{2} \mathrm{O}-\mathrm{HF}$}

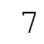

6

8

8

8

6

6

1

1

6

1

1

1

7

1

9

$$
\begin{aligned}
& 1 . 47022 \\
& 1.60261 \\
& 2.70227 \\
& 1.71935 \\
&-1.86263 \\
&-0.87187 \\
&-0.90961 \\
&-1.87333 \\
& 0.2975 \\
& 0.25438 \\
& 2.3394 \\
& 0.4823 \\
& 2.46686 \\
& 1.92476 \\
& 0.40185 \\
&-3.28249 \\
&-4.04273
\end{aligned}
$$

-0.07591
0.00235
0.03927
0.3057
0.01592
-0.01235
-0.09217
-0.12757
-0.1787
-0.12006
-0.09597
0.0959
0.19065
-0.17574
0.03234

$-0.05007$

$-0.10081$

$$
\begin{array}{r}
-1.50249 \\
-0.12343 \\
0.42179 \\
3.04558 \\
0.73555 \\
-0.00581 \\
-1.45608 \\
-1.94626 \\
-3.22569 \\
-2.14408 \\
-2.01853 \\
1.57993 \\
2.43167 \\
3.85523 \\
0.55698 \\
-0.00112 \\
-0.58929
\end{array}
$$

\section{HF-NH3}
1. 42103
0.02774
$-1.40381$
1.5867
0.00286
$-0.03707$ 


$\begin{array}{rcr}2.71545 & -0.00553 & 0.47412 \\ 2.27345 & -0.05116 & 2.96604 \\ -1.85062 & -0.0169 & 0.92729 \\ -0.89472 & -0.00261 & 0.16205 \\ -0.96281 & 0.02377 & -1.29493 \\ -1.94735 & 0.03083 & -1.75177 \\ 0.19725 & 0.0575 & -3.09655 \\ 0.18022 & 0.03778 & -2.01293 \\ 2.27475 & 0.03853 & -1.94495 \\ 0.5207 & -0.0297 & 1.69836 \\ 2.63644 & -0.03464 & 2.07257 \\ 0.41965 & -0.01141 & 0.68309 \\ -3.80731 & -0.00366 & -0.24471 \\ -4.26576 & 0.01105 & -1.15623 \\ -4.86399 & -0.80885 & -1.21634 \\ -4.87093 & 0.82748 & -1.18645\end{array}$

\section{$\mathrm{HF}-\mathrm{H}_{2} \mathrm{O}$}

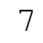

6

8

\section{HF-HF}

$$
\begin{gathered}
1.4722 \\
1.56342 \\
2.65909 \\
2.05333 \\
-1.91487 \\
-0.9182 \\
-0.90974 \\
-1.86026 \\
0.34355 \\
0.26942 \\
2.3533 \\
0.40375 \\
2.48422 \\
0.35818 \\
-3.53947 \\
-4.05949 \\
-4.98501
\end{gathered}
$$

$$
\begin{array}{r}
1.50675 \\
1.55538 \\
2.62991 \\
1.90056 \\
-1.94581 \\
-0.92187 \\
-0.87337 \\
-1.80753 \\
0.43423 \\
0.32752 \\
2.40444 \\
0.33918 \\
2.39238 \\
0.32562 \\
-3.34367 \\
-4.03883
\end{array}
$$

$$
\begin{array}{cc}
-0.07175 & -1.37529 \\
-0.07257 & -0.00081 \\
-0.12132 & 0.57279 \\
-0.08636 & 3.0358 \\
0.08876 & 0.77792 \\
0.04147 & 0.06206 \\
0.03797 & -1.39465 \\
0.08113 & -1.91146 \\
-0.02362 & -3.13198 \\
-0.01784 & -2.05075 \\
-0.11329 & -1.86945 \\
-0.01552 & 1.67428 \\
-0.10869 & 2.1747 \\
-0.01595 & 0.65434 \\
0.16077 & -0.25388 \\
0.18337 & -1.07925 \\
0.2696 & -0.82457
\end{array}
$$

$$
\begin{array}{rr}
0.01245 & -1.37939 \\
-0.00264 & -0.0014 \\
-0.01146 & 0.60763 \\
-0.03656 & 3.04615 \\
-0.00407 & 0.66966 \\
0.00221 & -0.01908 \\
0.01809 & -1.47095 \\
0.02574 & -2.01678 \\
0.03358 & -3.17015 \\
0.02251 & -2.09171 \\
0.01574 & -1.84556 \\
-0.01789 & 1.63705 \\
-0.02856 & 2.22094 \\
-0.0065 & 0.61522 \\
0.00689 & -0.16745 \\
0.01502 & -0.82707
\end{array}
$$

\section{S14}




\section{Uracil Anion-Water Complexes}

\section{O2(N3)}

7

6

8

8

8

6

6

1

1

6

1

1

1

\section{O4(N3)}

8

6

6

1

1

6

6

6
8

\section{O4(C5)}

1

6

6

1

$$
\begin{array}{ll}
\multicolumn{1}{l}{0 .} & \multicolumn{1}{l}{0 .} \\
0 . & 0 . \\
1.04833 & 0 . \\
0.78521 & 0.32402 \\
-3.52549 & 0.01561 \\
-2.50731 & 0.00922 \\
-2.44538 & 0.00869 \\
-3.36924 & 0.01162 \\
-1.15943 & 0.00134 \\
-1.20345 & 0.00289 \\
-1.20538 & -0.00145 \\
0.94778 & 0.15155 \\
0.89742 & 1.27671 \\
-1.23442 & 0.00209
\end{array}
$$

0 .

1.35648

2.05128

4.70788

2.15374

1.4369

0.00296

$-0.56311$

$-1.69674$

$-0.60473$

3.04037

3.73927

4.80369

2.02529

$$
\begin{array}{ccr}
0.67105 & 0.07945 & \multicolumn{1}{c}{-1.91098} \\
0.68164 & 0.06917 & -0.65019 \\
1.83288 & 0.05217 & 0.18888 \\
2.81801 & 0.05064 & -0.26114 \\
2.52306 & 0.02538 & 2.20818 \\
1.63908 & 0.03847 & 1.56471 \\
0.47295 & 0.03801 & 2.22286 \\
-0.66898 & 0.0555 & 1.46737 \\
-1.82189 & 0.05767 & 1.92375 \\
-0.5141 & 0.07116 & 0.05833 \\
-1.37437 & 0.09398 & -0.47817 \\
-1.64618 & -1.21622 & -3.40575 \\
-1.61976 & -0.25488 & -3.33852 \\
-0.77541 & -0.06776 & -2.85008
\end{array}
$$




\section{Uracil Anion-Ammonia Complexes}

\section{O2(N3)}

7
6
8
7
1
8
6
6
1
1
6
1
1
1
7

\section{O4(N3)}

1

1

7

1

8

6

7

1

8

6

7

1

6

6

1

\section{4(C5)}

1

$\begin{array}{rrr}0.40719 & -1.70926 & -0.00137 \\ -0.40633 & -0.61737 & -0.04656 \\ -1.6536 & -0.67004 & -0.09376 \\ -4.37626 & 0.64175 & 0.0842 \\ -4.82331 & 0.34952 & -0.78178 \\ 1.96026 & 2.12428 & 0.00069 \\ 1.57087 & 0.93971 & 0.00315 \\ 2.37183 & -0.2491 & 0.04728 \\ 3.45085 & -0.15721 & 0.08339 \\ 2.34637 & -2.38013 & 0.07691 \\ 1.73127 & -1.47687 & 0.04217 \\ -0.42571 & 1.44913 & -0.07229 \\ -3.40572 & 0.31073 & 0.03605 \\ -4.82042 & 0.11409 & 0.83223 \\ 0.20033 & 0.65347 & -0.04086\end{array}$

$-4.81141$

$-4.8934$

$-4.41911$

$-3.4405$

$-1.68042$

$-0.42832$

0.20126

$-0.39693$

1. 96413

1. 59313

2.42934

2.56076

1.85499

0.49825

0.12303

0.25752

0.16817

0.63633

0.32907

$-0.66377$

$-0.59301$

0.6504

1. 46732

2. 09257

0.90775

$-0.17528$

$-2.22186$

$-1.38681$

$-1.6803$

$-2.69658$

0.89396

$-0.73364$

0.03508

0.00206

$-0.05682$

$-0.02901$

$-0.02652$

$-0.05018$

$-0.00266$

0.00146

0.03131

0.05388

0.02992

0.0022

0.00316

$\begin{array}{rr}0.0006 & -1.88719 \\ -0.00169 & -0.82261 \\ -0.01129 & 0.12619 \\ -0.01672 & -0.22867 \\ -0.01488 & 1.46139 \\ -0.00837 & 1.96218 \\ -0.0105 & 3.16993 \\ 0.0015 & 1.02716 \\ 0.00621 & 1.41837 \\ 0.00543 & -0.36311 \\ 0.01436 & -1.05418 \\ 0.01738 & -3.03456 \\ 0.01784 & -3.98808 \\ -0.80297 & -4.05973 \\ 0.82899 & -4.05338\end{array}$




\section{Uracil Anion-Hydrogen Fluoride Complexes}

\section{O2(N3)}

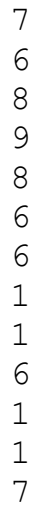

\section{O4(N3)}

\section{O4(C5)}

\section{Uracil Anion 02(N3)-O4(N3) Complexes}

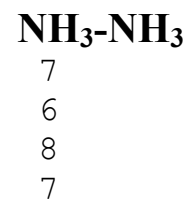

$\begin{array}{ccc}0.4449 & -1.77601 & -0.00141 \\ -0.50862 & -0.82447 & 0.00116 \\ -1.7486 & -1.10111 & 0.00268 \\ -3.18198 & 0.83368 & -0.00263 \\ 1.3778 & 2.24233 & -0.00023 \\ 1.1755 & 1.01628 & 0.00095 \\ 2.15624 & -0.03362 & -0.00039 \\ 3.20667 & 0.23161 & -0.00036 \\ 2.46946 & -2.1418 & -0.00317 \\ 1.7238 & -1.34396 & -0.0016 \\ -0.88701 & 1.20714 & 0.00222 \\ -2.61579 & -0.02169 & -0.0002 \\ -0.13784 & 0.51979 & 0.00209\end{array}$

$-0.81502$

1.25518

0.52994

0.98372

2.20514

0.02809

$-1.25002$

$-1.97579$

$-2.79108$

$-1.73365$

$-0.78624$

$-1.06$

0.01194

0.79438

0.003

0.00298

0.00056

0.00136

$-0.00227$

$-0.00305$

$-0.00558$

$-0.00181$

$-0.00102$

0.00238

0.00444

0.01323

$-0.00594$

$\begin{array}{rcc}0.81392 & -0.00257 & -2.06802 \\ 0.73482 & -0.00192 & -0.98849 \\ 1.84857 & -0.00314 & -0.1532 \\ 2.83732 & -0.00438 & -0.61837 \\ 1.86332 & -0.00191 & 1.18419 \\ 0.65208 & 0.00044 & 1.82315 \\ 0.49312 & 0.0015 & 3.05223 \\ -0.50493 & 0.00149 & 1.00601 \\ -1.39332 & 0.00316 & 1.49191 \\ -0.54368 & 0.00016 & -0.37548 \\ -1.67965 & 0.00181 & -0.94281 \\ -1.76869 & 0.00114 & -2.38605 \\ -1.85072 & 0.00065 & -3.38467\end{array}$


$-0.08236$

$-0.96544$

$-0.56922$

$-0.60321$

$-1.01078$

$-0.13838$

$-0.11178$

0.0465

0.79031

1. 48801

$-0.01649$

$-0.71361$

$-0.37666$

0.46638

$-0.08619$
3.25473

0.00686

$-0.70368$

$-2.1329$

$-2.70745$

$-3.81903$

$-2.72839$

0.90731

1.86998

3. 35611

$-0.10574$

2. 25099

3.1958

3.3232

3.19841

\section{$\mathrm{NH}_{3}-\mathrm{H}_{\mathbf{2}} \mathrm{O}$}

$$
\begin{aligned}
& 0.80715 \\
& 1.07757 \\
& 2.19732 \\
& 2.28229 \\
& 2.6543
\end{aligned}
$$

$-2.17843$

$-1.32129$

$-1.5476$

$-2.54496$

$-0.63191$

$-0.46083$

$-1.5732$

$-1.08313$

$-1.41587$

\section{$\mathbf{N H}_{3}-\mathbf{H F}$}

0.22471

2.39674

1.28136

0.00137

$$
\begin{array}{r}
-0.34725 \\
-0.29772 \\
-0.48535 \\
0.06231 \\
1.00716 \\
0.45064 \\
0.21565 \\
0.1494 \\
0.31363 \\
-0.17603 \\
-0.12645 \\
0.02643 \\
-0.23602 \\
0.12969 \\
-0.01235 \\
0.36827 \\
0.23484 \\
-0.59855
\end{array}
$$

$$
\begin{aligned}
& -2.27794 \\
& -0.9386 \\
& -0.43147 \\
& 2.81175 \\
& 2.86899 \\
& 0.47387 \\
& -0.42419 \\
& -1.82797 \\
& -2.21676 \\
& -3.72833 \\
& -2.64979 \\
& 0.91921 \\
& 1.83977 \\
& 2.99832 \\
& -0.06943 \\
& 2.06823 \\
& 2.9279 \\
& 3.28068
\end{aligned}
$$

$\begin{array}{rcc}0.94889 & 0.14861 & -1.98907 \\ 0.97833 & 0.12222 & -0.62316 \\ 2.00946 & 0.21323 & 0.0621 \\ 3.77225 & 0.40702 & 2.60456 \\ 3.10038 & 0.42256 & 3.36839 \\ -2.53797 & -0.2684 & 0.18984 \\ -1.50103 & -0.14093 & -0.53477 \\ -1.48083 & -0.10619 & -1.95144 \\ -2.40962 & -0.19151 & -2.50082 \\ -0.22221 & 0.06348 & -3.66894 \\ -0.24712 & 0.03675 & -2.57692 \\ -0.21149 & -0.04199 & 1.06827 \\ 3.22906 & 0.46088 & 1.73888 \\ 4.20412 & -0.51399 & 2.61406 \\ -0.25561 & -0.02213 & 0.05394 \\ -2.33107 & -0.28986 & 1.59351 \\ -2.09843 & -0.2971 & 2.57809\end{array}$




\section{$\mathrm{H}_{2} \mathrm{O}-\mathrm{NH}_{3}$}

7
6
8
8
8
6
6
1
1
6
1
1
1
7
1
7
1
1

$\begin{array}{ccc}2.32267 & -0.61387 & 0.20832 \\ 1.35879 & 0.33796 & 0.16843 \\ 1.58393 & 1.57048 & 0.29894 \\ -0.64642 & 3.01255 & -0.00528 \\ -1.65314 & -1.5793 & -0.37302 \\ -0.43434 & -1.37196 & -0.19973 \\ 0.62282 & -2.33857 & -0.14637 \\ 0.38501 & -3.38891 & -0.26411 \\ 2.72134 & -2.6264 & 0.09127 \\ 1.9162 & -1.88883 & 0.05233 \\ -0.66541 & 0.67391 & -0.05603 \\ 0.23592 & 2.56522 & 0.15391 \\ -0.55272 & 3.45874 & -0.8548 \\ 0.03075 & -0.06412 & -0.03046 \\ -3.04866 & 0.28178 & -0.20763 \\ -3.44983 & 1.19037 & 0.03574 \\ -2.6887 & 1.87047 & 0.03006 \\ -3.76764 & 1.11708 & 0.99912\end{array}$

\section{$\mathrm{H}_{2} \mathrm{O}-\mathrm{H}_{2} \mathrm{O}$}

7
6
8
8
8
6
6
1
1
6
1
1
1
7
1
8
1

$$
\begin{array}{r}
1.31917 \\
1.27235 \\
2.28772 \\
1.42055 \\
-2.26496 \\
-1.22739 \\
-1.1275 \\
-2.03064 \\
0.21267 \\
0.13512 \\
0.02617 \\
1.88064 \\
1.62463 \\
0.01759 \\
-2.01838 \\
-1.76441 \\
-0.79916
\end{array}
$$

$$
\begin{array}{rr}
0.03027 & -2.00184 \\
0.05946 & -0.64798 \\
0.10228 & 0.09682 \\
0.04133 & 2.63721 \\
-0.06408 & 0.05435 \\
-0.03361 & -0.64622 \\
-0.06706 & -2.07334 \\
-0.12293 & -2.66866 \\
-0.053 & -3.73099 \\
-0.02985 & -2.64162 \\
0.07988 & 0.98781 \\
0.03406 & 1.74661 \\
0.90085 & 3.02395 \\
0.04256 & -0.02586 \\
-0.13234 & 2.00484 \\
-0.14786 & 2.95176 \\
-0.24337 & 2.96423
\end{array}
$$

\section{$\mathrm{H}_{2} \mathrm{O}-\mathrm{HF}$}

$-0.29988$

$-1.9836$

$-0.25994$

$-2.54366$

$-0.18607$

$-3.68056$

0.06563

$-2.59176$

0.27598

1.04382

0.3222

1.87701

$-0.00537$

3.18198

0.03089

$-0.01037$

1.52784

0.07462

2.49123 


\section{HF- $\mathrm{NH}_{3}$}

7

6

8

9

8

6

6

1

1

6

1

1

7

1

7

1

1

\section{HF- $\mathrm{H}_{2} \mathrm{O}$}

6

8

9

8

6

6

1

1

6

1

1

7

1

8

1

\section{HF-HF}

$$
\begin{array}{ccc}
-2.163 & 0.97081 & 0.09102 \\
-0.82673 & 1.11541 & 0.02961 \\
-0.25621 & 2.25254 & 0.0373 \\
2.12508 & 2.02444 & -0.13291 \\
0.37864 & -2.2702 & -0.14906 \\
-0.45242 & -1.34491 & -0.07398 \\
-1.88129 & -1.45436 & -0.00736 \\
-2.33378 & -2.43842 & -0.02114 \\
-3.71639 & -0.37759 & 0.12152 \\
-2.6289 & -0.2962 & 0.07056 \\
0.98757 & 0.14466 & -0.08327 \\
1.10465 & 2.19463 & -0.05775 \\
-0.01583 & -0.01472 & -0.04575 \\
2.57935 & -1.75593 & -0.07174 \\
3.47298 & -1.29913 & 0.1243 \\
3.41186 & -0.3471 & -0.23337 \\
3.53843 & -1.21724 & 1.13603
\end{array}
$$

$$
\begin{array}{cc}
0.10851 & -1.99957 \\
0.05103 & -0.65521 \\
0.04406 & 0.03678 \\
-0.06559 & 2.37325 \\
-0.05798 & 0.17103 \\
-0.0057 & -0.55021 \\
0.05635 & -1.9795 \\
0.06064 & -2.5332 \\
0.15621 & -3.68908 \\
0.10875 & -2.59924 \\
-0.04655 & 1.04406 \\
-0.01761 & 1.39156 \\
-0.00402 & 0.02969 \\
-0.11832 & 2.01536 \\
-0.148 & 2.99773 \\
-0.14267 & 3.26189
\end{array}
$$

$\begin{array}{rr}0.00259 & -1.98578 \\ 0.00064 & -0.63656 \\ -0.00048 & 0.01066 \\ -0.00612 & 2.43075 \\ -0.00026 & 0.25517 \\ 0.00073 & -0.47358 \\ 0.00278 & -1.89727 \\ 0.00364 & -2.41983 \\ 0.00516 & -3.64547 \\ 0.00356 & -2.55405 \\ -0.00174 & 1.10143 \\ -0.00379 & 1.43744 \\ -0.00014 & 0.08617 \\ -0.00067 & 1.74609 \\ -0.00056 & 2.71588\end{array}$ 


\section{Uracil Anion O4(N3)-O4(C5) Complexes}

$\begin{array}{cccc}\mathbf{N H}_{\mathbf{3}} \mathbf{N H}_{\mathbf{3}} & & & \\ 6 & -0.1891 & -0.03132 & 0.21426 \\ 6 & -0.15352 & -0.18751 & 1.62927 \\ 6 & 1.08708 & -0.17525 & 2.25604 \\ 7 & 2.28857 & -0.02883 & 1.68321 \\ 6 & 2.3153 & 0.12736 & 0.32337 \\ 7 & 1.07009 & 0.11824 & -0.35273 \\ 8 & 3.34234 & 0.27546 & -0.35578 \\ 8 & -1.21126 & -0.0177 & -0.52484 \\ 7 & -4.16246 & 0.11073 & 0.23996 \\ 1 & -1.08009 & -0.31513 & 2.17534 \\ 1 & 1.11432 & -0.29732 & 3.34209 \\ 1 & 1.11408 & 0.23616 & -1.35806 \\ 1 & -3.15008 & 0.02314 & 0.08902 \\ 1 & -4.38778 & 1.09909 & 0.1553 \\ 1 & -4.61729 & -0.36353 & -0.5365 \\ 1 & -1.51493 & -0.10845 & -2.52254 \\ 7 & -1.79374 & -0.19548 & -3.50649 \\ 1 & -2.61487 & 0.3902 & -3.63635 \\ 1 & -2.08784 & -1.15855 & -3.64867\end{array}$

\section{$\mathrm{NH}_{3}-\mathrm{H}_{2} \mathrm{O}$}

$-0.06582$

$-0.18535$

$-0.09058$

0.11728

0.98475

0.22687

1.49839

2.24269

2.41624

0.44307

2.21912

0.38749

1.76611

1.24705

0.08468

0.44215

3.51157

$-0.14103$

$-0.00661$

$-0.32547$

$-0.99832$

$-0.32103$

$-3.64749$

$-0.62208$

$-1.16736$

0.299

0.68911

$-0.13097$

$-0.70432$

$-0.1752$

0.89502

1.39543

$-0.3671$

1.94921

3.28028

$-2.67497$

$-0.45887$

$-1.30138$

$-3.71725$

$-1.57674$

$-0.29499$

$-2.00956$

0.11675

$-0.06052$

$-2.91532$

0.26442

$-2.77453$

$-3.5901$

0.07728

$-3.22126$

$-2.97727$

1.25794

$-2.47941$

$-3.43196$

$\mathbf{N H}_{\mathbf{3}}-\mathbf{H F}$
6
6
6
7
6
7
8
8
9
1
1

$$
\begin{aligned}
& -0.34137 \\
& -1.56754 \\
& -1.50776 \\
& -0.41274 \\
& 0.79979 \\
& 0.77926 \\
& 1.90102 \\
& -0.1806 \\
& -2.12172 \\
& -2.50252 \\
& -2.44983
\end{aligned}
$$




$\begin{array}{rcr}0.16091 & 1.6842 & -0.02089 \\ -2.32196 & -1.3478 & -0.00559 \\ -2.54532 & 1.5403 & 0.01187 \\ -3.09983 & 2.40164 & 0.02057 \\ -3.68716 & 2.36866 & 0.85024 \\ -3.72199 & 2.36325 & -0.78305\end{array}$

\section{$\mathrm{H}_{2} \mathrm{O}-\mathrm{NH}_{3}$}

$\begin{array}{rrr}0.20828 & -0.27831 & -0.10323 \\ -0.28177 & -1.60009 & -0.27908 \\ -1.65792 & -1.79639 & -0.22406 \\ -2.60294 & -0.8722 & -0.02065 \\ -2.18551 & 0.42044 & 0.15626 \\ -0.7886 & 0.66147 & 0.10773 \\ -2.93344 & 1.3879 & 0.35391 \\ 1.4208 & 0.08615 & -0.12451 \\ 4.06392 & -1.43984 & 0.27642 \\ 0.41535 & -2.40959 & -0.45601 \\ -2.03741 & -2.81235 & -0.36051 \\ -0.50299 & 1.62415 & 0.25013 \\ 3.14624 & -1.03676 & 0.05916 \\ 4.72079 & -0.66282 & 0.28644 \\ 4.00912 & -1.78567 & 1.23174 \\ 1.90931 & 1.75393 & -0.10714 \\ 2.13955 & 2.71538 & -0.18173 \\ 2.11923 & 2.89366 & -1.129\end{array}$

\section{$\mathrm{H}_{2} \mathrm{O}-\mathrm{H}_{2} \mathrm{O}$}

$$
\begin{array}{rr}
-0.2865 & -0.26889 \\
0.17052 & -1.60712 \\
1.54641 & -1.82141 \\
2.50985 & -0.89907 \\
2.11856 & 0.41208 \\
0.72345 & 0.6712 \\
2.88753 & 1.37924 \\
-1.49887 & 0.11871 \\
-3.72364 & -1.50834 \\
-0.546 & -2.41614 \\
1.90562 & -2.85169 \\
0.45459 & 1.64785 \\
-2.89634 & -0.97669 \\
-4.10128 & -1.19917 \\
-1.87194 & 1.81729 \\
-1.99334 & 2.79942 \\
-2.24507 & 2.97356
\end{array}
$$$$
-1.60712
$$

$-1.82141$

$-0.89907$

0.41208

0.6712

1. 37924

0.11871

$-1.50834$

$-2.41614$

$-2.85169$

1. 64785

$-0.97669$

$-1.19917$

1.81729

2.79942

2.97356

0.02347

0.1061

0.09853

0.02352

$-0.0566$

$-0.0568$

$-0.12812$

0.01862

$-0.05606$

0.17087

0.16067

$-0.12051$

0.03974

$-0.88749$

$-0.01371$

0.0294

0.9436

\section{$\mathrm{H}_{2} \mathrm{O}$-HF}

$-0.35989$

$-0.27822$

$-0.0438$

0.08461

$-1.61598$

$1.46208-1.83068$

0.01524

2.42366

$-0.90683$

0.05383

2.0352

0.40791

0.6402

0.66717

0.04497

2.80538

1.37462

$-0.00853$

$-1.57843$

0.11933

$-0.0566$

$-0.0171$

$-0.08384$ 


$\begin{array}{rrr}-3.42945 & -1.5066 & 0.01437 \\ -0.63481 & -2.42436 & 0.02809 \\ 1.81934 & -2.8623 & 0.09733 \\ 0.36841 & 1.64555 & -0.09759 \\ -2.67505 & -0.86352 & -0.03254 \\ -1.86374 & 1.86412 & -0.08739 \\ -1.82748 & 2.84813 & -0.01613 \\ -2.1239 & 3.04282 & 0.88036\end{array}$

\section{$\mathrm{HF}-\mathrm{NH}_{3}$}

6
6
6
7
6
7
8
8
7
1
1
1
1
1
1
1
9

-0.30294
-0.38138
0.81702
2.05408
2.18022
0.98039
3.25378
-1.29806
-4.35458
-1.34923
0.76671
1.09566
-3.33102
-4.6646
-4.66789
-1.0317
-0.78894

$\begin{array}{cc}0.00066 & 0.13401 \\ 0.00036 & 1.54528 \\ -0.00255 & 2.25591 \\ -0.00513 & 1.75465 \\ -0.00496 & 0.38936 \\ -0.00204 & -0.37018 \\ -0.00714 & -0.22422 \\ 0.00315 & -0.66717 \\ 0.00978 & -0.18961 \\ 0.0023 & 2.02989 \\ -0.0028 & 3.3475 \\ -0.00197 & -1.37876 \\ 0.00773 & -0.23235 \\ 0.82687 & -0.71034 \\ -0.80503 & -0.71196 \\ 0.0029 & -2.07376 \\ 0.00251 & -3.05005\end{array}$

\section{HF- $\mathrm{H}_{2} \mathrm{O}$}

$-0.03408$

$-0.0168$

0.12829

0.25486

0.24342

0.09724

0.34992

$-0.15843$

$-0.45663$

$-0.11416$

0.14302

0.09125

$-0.34832$

$-0.50795$

$-0.15755$

$-0.15277$

0.1622

1.57244

2.17998

1.5759

0.20469

$-0.45052$

$-0.49773$

$-0.55721$

0.18487

2.13765

3.27165

$-1.46498$

0.03401

$-0.70515$

$-1.9981$

$-2.98978$

\section{HF-HF}

$$
\begin{gathered}
-0.40766 \\
-0.42243 \\
0.8146 \\
2.01975 \\
2.07612 \\
0.83622 \\
3.11399 \\
-1.44575
\end{gathered}
$$

0.00014

0.00029

0.00006

$-0.00028$

$-0.00035$

0.00005

$-0.0007$

0.00016 


$\begin{array}{rrrr}9 & -3.72415 & 0.00123 & 0.35107 \\ 1 & -1.36409 & 0.0004 & 2.04331 \\ 1 & 0.82125 & 0.00015 & 3.25065 \\ 1 & 0.89474 & 0.00085 & -1.48825 \\ 1 & -2.82788 & 0.00082 & -0.04943 \\ 1 & -1.21354 & -0.00047 & -2.12733 \\ 9 & -0.94384 & -0.00086 & -3.07841\end{array}$

\section{Uracil Anion 02(N3)-O4(C5) Complexes}

\section{$\mathrm{NH}_{3}-\mathrm{NH}_{3}$}

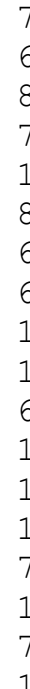

6

8

7

1

8

6

1

1

6

1

1

7

1

7

1

\section{$\mathrm{NH}_{3}-\mathrm{H}_{2} \mathrm{O}$}

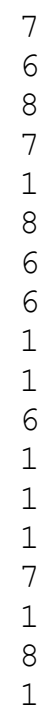

\section{$\mathrm{NH}_{3}-\mathrm{HF}$}

$$
\begin{gathered}
1.82281 \\
1.71451 \\
2.6947 \\
1.88604 \\
2.22221 \\
-1.86703 \\
-0.78678 \\
-0.61436 \\
-1.48897 \\
0.8081 \\
0.6759 \\
0.36617 \\
2.31348 \\
2.21699 \\
0.4225 \\
-3.8032 \\
-4.82672 \\
-5.16322 \\
-5.16098
\end{gathered}
$$

0.02957

0.0089

0.001

$-0.04548$

$-0.87239$

$-0.01222$

0.00133

0.0231

0.02898

0.05214

0.03554

$-0.01972$

$-0.0292$

0.76764

$-0.00432$

$-0.01234$

$-0.01146$

$-0.82407$

0.80738

$-0.02102$

$-0.0227$

$-0.01383$

$-0.01137$

$-0.83139$

$-0.05623$

$-0.04532$

$-0.04238$

$-0.04793$

$-0.02834$

$-0.03051$

$-0.03552$

$-0.01143$

0.80973

$-0.03495$

0.01833

0.15544

0.76031
$-1.54006$

$-0.18291$

0.5957

3. 41849

3. 90484

0.31738

$-0.31692$

$-1.7368$

$-2.37598$

$-3.32521$

$-2.24031$

1. 39454

2.4807

3. 93097

0.379

$-0.24288$

$-0.3363$

0.17531

0.16676

$$
\begin{gathered}
-1.58963 \\
-0.22822 \\
0.4997 \\
3.3282 \\
3.83642 \\
0.45338 \\
-0.24035 \\
-1.66411 \\
-2.25841 \\
-3.32231 \\
-2.23223 \\
1.41589 \\
2.39962 \\
3.83558 \\
0.39767 \\
-0.13117 \\
-0.31788 \\
0.3785
\end{gathered}
$$

$\begin{array}{lrr}1.5595 & 0.01739 & -1.64185 \\ 1.60766 & 0.00092 & -0.27975 \\ 2.66805 & -0.00854 & 0.38076\end{array}$




$\begin{array}{ccc}1.93599 & -0.04016 & 3.21718 \\ 2.22661 & -0.86735 & 3.73076 \\ -1.88343 & -0.00489 & 0.63045 \\ -0.87616 & 0.00286 & -0.14004 \\ -0.88216 & 0.01999 & -1.56068 \\ -1.82531 & 0.02778 & -2.09201 \\ 0.35338 & 0.03912 & -3.3013 \\ 0.34537 & 0.02599 & -2.20891 \\ 0.4431 & -0.01815 & 1.44361 \\ 2.4193 & -0.03044 & 2.30842 \\ 2.22755 & 0.77524 & 3.74877 \\ 0.38514 & -0.00586 & 0.42514 \\ -3.20993 & 0.0032 & 0.06335 \\ -4.13827 & 0.00889 & -0.3176\end{array}$

\section{$\mathrm{H}_{2} \mathrm{O}-\mathrm{NH}_{3}$}

$$
\begin{array}{r}
1.84754 \\
1.69371 \\
2.65468 \\
1.97596 \\
-1.89484 \\
-0.79864 \\
-0.58133 \\
-1.43545 \\
0.89025 \\
0.72272 \\
0.30284 \\
2.30894 \\
2.13177 \\
0.39032 \\
-3.80981 \\
-4.82417 \\
-5.18967 \\
-5.19981
\end{array}
$$

\section{$\mathrm{H}_{2} \mathrm{O}-\mathrm{H}_{2} \mathrm{O}$}

$$
\begin{array}{rr}
-0.00427 & -1.43457 \\
-0.06458 & -0.08808 \\
-0.11531 & 0.72053 \\
0.01445 & 3.31554 \\
-0.01752 & 0.30895 \\
-0.01119 & -0.29445 \\
0.05094 & -1.70715 \\
0.09774 & -2.37165 \\
0.09542 & -3.24936 \\
0.04874 & -2.1708 \\
-0.11643 & 1.4473 \\
-0.08099 & 2.3803 \\
0.93983 & 3.53633 \\
-0.06876 & 0.43594 \\
0.03817 & -0.34544 \\
0.05141 & -0.50659 \\
-0.81943 & -0.12826 \\
0.80065 & 0.07012
\end{array}
$$

\begin{tabular}{rrr}
1.73964 & -0.02792 & \multicolumn{1}{c}{-1.4746} \\
1.63946 & -0.07669 & -0.12223 \\
2.6305 & -0.09934 & 0.64884 \\
2.00404 & 0.03248 & 3.25627 \\
-1.92782 & -0.09471 & 0.41569 \\
-0.85393 & -0.07386 & -0.2357 \\
-0.69766 & -0.02291 & -1.65329 \\
-1.57741 & 0.00136 & -2.28444 \\
0.7129 & 0.03362 & -3.25201 \\
0.58831 & -0.00483 & -2.16756 \\
0.30801 & -0.13883 & 1.46633 \\
2.32948 & -0.05819 & 2.3184 \\
2.17143 & 0.95344 & 3.48676 \\
0.35653 & -0.10122 & 0.45153 \\
-3.55109 & 0.03949 & -0.25531 \\
-4.49567 & 0.20536 & -0.50087 \\
-4.81199 & 0.79253 & 0.19508
\end{tabular}




\section{$\mathrm{H}_{2} \mathrm{O}-\mathrm{HF}$}

\section{HF- $\mathrm{NH}_{3}$}

\section{HF- $\mathrm{H}_{2} \mathrm{O}$}

7

$\begin{array}{ccc}1.61004 & 0.00579 & -1.52349 \\ 1.58492 & -0.05467 & -0.16743 \\ 2.61447 & -0.09378 & 0.5477 \\ 2.03333 & -0.00683 & 3.16951 \\ -1.94107 & -0.03899 & 0.57075 \\ -0.90041 & -0.02408 & -0.14996 \\ -0.8322 & 0.0392 & -1.5681 \\ -1.74691 & 0.07774 & -2.14567 \\ 0.49106 & 0.0955 & -3.24159 \\ 0.42568 & 0.04812 & -2.15265 \\ 0.33892 & -0.11636 & 1.49191 \\ 2.36518 & -0.07427 & 2.23307 \\ 2.2183 & 0.9009 & 3.4363 \\ 0.33334 & -0.07234 & 0.47525 \\ -3.24858 & 0.02387 & -0.06456 \\ -4.14926 & 0.06932 & -0.49406\end{array}$

1.85563

0.02551

0.00425

$-0.00642$

$-0.04442$

$-0.00964$

0.00215

0.02475

0.03325

0.05195

0.03476

$-0.02288$

$-0.02845$

$-0.00666$

$-0.00343$

$-0.00204$

$-0.824$

0.80873

$-1.35379$

$-0.0181$

0.7994

3.10357

0.35174

$-0.23983$

$-1.6532$

$-2.32456$

$-3.18309$

$-2.10647$

1. 51625

2.14432

0.50341

$-0.39972$

$-0.63729$

$-0.19676$

$-0.17835$

$\begin{array}{rrr}1.7537 & 0.00898 & -1.39029 \\ 1.62484 & -0.00536 & -0.04871 \\ 2.62034 & 0.01365 & 0.73598 \\ 1.97829 & -0.00616 & 3.06162 \\ -1.93504 & -0.09657 & 0.44396 \\ -0.85429 & -0.06492 & -0.1905 \\ -0.67722 & -0.04804 & -1.60802 \\ -1.549 & -0.06187 & -2.25031 \\ 0.75519 & 0.00038 & -3.18712 \\ 0.61225 & -0.01255 & -2.10513 \\ 0.28843 & -0.05277 & 1.53069 \\ 2.31389 & 0.00315 & 2.09752 \\ 0.3475 & -0.0423 & 0.51514 \\ -3.54067 & -0.01981 & -0.29908 \\ -4.46305 & 0.12847 & -0.62286 \\ -4.79237 & 0.8405 & -0.06267\end{array}$




\section{HF-HF}

7

6

8

9

8

6

6

1

1

6

1

1

7

1

9

$\begin{array}{ccc}-1.03064 & 1.92023 & -0.00031 \\ -1.43869 & 0.6349 & -0.00005 \\ -2.65951 & 0.3018 & 0.00008 \\ -2.94909 & -2.09724 & 0.00054 \\ 1.63824 & -1.21486 & 0.00015 \\ 0.8921 & -0.19603 & -0.00001 \\ 1.29128 & 1.1695 & -0.00028 \\ 2.34539 & 1.41566 & -0.00038 \\ 0.58878 & 3.18439 & -0.00063 \\ 0.29714 & 2.13294 & -0.00042 \\ -0.82199 & -1.34302 & 0.0003 \\ -2.90257 & -1.08135 & 0.00035 \\ -0.48103 & -0.38397 & 0.0001 \\ 3.09577 & -1.03351 & 0.00003 \\ 4.08193 & -0.90714 & -0.00004\end{array}$

